\title{
Poisson structures in BRST-antiBRST invariant Lagrangian formalism
}

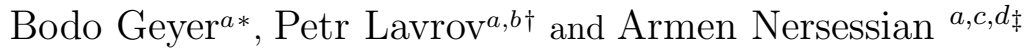 \\ a) Center of Theoretical Studies, Leipzig University, Augustusplatz 10/11, D-04109 Leipzig, Germany \\ b) Tomsk State Pedagogical University, 634041 Tomsk, Russia \\ c) Laboratory of Theoretical Physics, Joint Institute for Nuclear Research, Dubna, 141980 Russia \\ d) Yerevan State University, A. Manoogian St., 3, Yerevan, 375025, Armenia
}

\begin{abstract}
We show that the specific operators $V^{a}$ appearing in the triplectic formalism - the most general $S p(2)$ symmetric Lagrangian BRST quantization scheme - can be viewed as the anti-Hamiltonian vector fields generated by a second-rank irreducible $S p(2)$ tensor. This allows for an explicit realization of the "triplectic algebra" being constructed from an arbitrary Poisson bracket on the space of the fields only, equipped by the flat Poisson connection. We show that the whole space of fields and antifields can be equipped by an even supersymplectic structure, when this Poisson bracket is nondegenerate. This observation opens the possibility to provide the BRST/antiBRST path integral by a well-defined integration measure, as well as to establish a direct link between $S p(2)$ symmetric Lagrangian and Hamiltonian BRST quantization schemes.
\end{abstract}

Introduction. The Batalin-Vilkovisky (BV) formalism [1] of Lagrangian quantization of general gauge theories, since its introduction, attracts permanent interest due to its covariance, universality and mathematical elegance. Now, its area of physical applications is much wider than offered in the initial prescription. The BV-formalism is outstanding also from the formal mathematical point of view because it is formulated in terms of seemingly exotic objects: the antibracket (odd Poisson bracket) and the (related) second-order operator $\Delta$.

The study of the formal geometrical structure of the BV formalism, performed during the last ten years, allowed to introduce its interpretation in terms of more traditional mathematical objects [2, 3], as well as to find the unusual behaviour of the antibracket with respect to integration theory 佃. On the other hand, there exists an extended, $S p(2)$ symmetric (BRST/antiBRST invariant) version of the BV formalism [5], and its geometrized version known as "triplectic formalism" [6, ]] (see also [8, 9]). In addition, there exists also a $S p(2)$ invariant extension whose structure is characterized by the superalgebra $\operatorname{osp}(1,2)$ [10].

In the BV formalism the original set of "physical" fields $\phi^{A}$ (including ghosts, antighosts, Lagrangian multipliers etc.) is doubled by the "antifields" $\phi_{A}^{*}$ with opposite grading $\epsilon\left(\phi_{A}^{*}\right)=\epsilon\left(\phi^{A}\right)+1$. On this set of fields and antifields the nondegenerate antibracket and the corresponding $\Delta$-operator are defined. In the $S p(2)$ symmetric versions of the BV-formalism the initial set of fields $\phi^{A}$ is extended by additional auxiliary fields $\bar{\phi}_{A}$ as well as the $S p(2)$ doublets $\phi_{A a}^{*}$ and $\pi^{A a}(a=1,2)$ of antifields with gradings $\epsilon\left(\bar{\phi}_{A}\right)=\epsilon\left(\phi^{A}\right)$, $\epsilon\left(\phi_{A a}^{*}\right)=\epsilon\left(\pi^{A a}\right)=\epsilon\left(\phi^{A}\right)+1$; the antifields $\pi^{A a}$ are used in the formulation of gauge fixing conditions. As usual, the $S p(2)$ indices are raised and lowered by the help of $\varepsilon^{a b}$ and $\varepsilon_{a b}: \varepsilon^{a c} \varepsilon_{c b}=\delta_{b}^{a}, \varepsilon_{a b}=-\varepsilon_{b a}, \varepsilon_{12}=1$.

On this extended space of fields, $\mathcal{M}$, the basic ingredients of the $S p(2)$ symmetric Lagrangian quantization schemes are formulated by pairs of $\Delta$-operators, $\Delta^{a}$, and odd vector fields, $V^{a}$, satisfying the following consistency conditions:

$$
\begin{gathered}
\Delta^{\{a} \Delta^{b\}}=0, \quad V^{\{a} V^{b\}}=0, \quad \epsilon\left(V^{a}\right)=\epsilon\left(\Delta^{a}\right)=1, \\
\Delta^{\{a} V^{b\}}+V^{\{a} \Delta^{b\}}=0 .
\end{gathered}
$$

Here and in the following the curly bracket denotes symmetrization with respect to the indices $a$ and $b$. These operators are formulated in terms of $\phi^{A}, \bar{\phi}_{A}, \phi_{A a}^{*}$ fields in the original scheme [5, and on the

*e-mail: geyer@itp.uni-leipzig.de

$\dagger$ e-mail: lavrov@tspu.edu.ru

‡e-mail: nerses@thsun1.jinr.ru 
whole set $z=\left\{\phi^{A}, \bar{\phi}_{A}, \phi_{A a}^{*}, \pi^{A a}\right\}$ in the case of triplectic formalism [6, 7]. An essential difference between the $S p(2)$ symmetric quantization schemes and the standard BV formalism is not only the doubling of $\Delta$-operators and corresponding antibrackets, but also the appearance of the vector fields $V^{a}$ and the degeneracy of the antibrackets generated by the $\Delta^{a}$-operators as obstruction of the Leibniz rule,

$$
(-1)^{\epsilon(f)}(f, g)^{a}=\Delta^{a}(f g)-\left(\Delta^{a} f\right) g-(-1)^{\epsilon(f)} f\left(\Delta^{a} g\right) .
$$

These antibrackets obey the conditions [5]:

$$
\begin{aligned}
& (f, g)^{a}=-(-1)^{(\epsilon(f)+1)(\epsilon(g)+1)}(g, f)^{a}, \\
& (-1)^{(\epsilon(f)+1)(\epsilon(h)+1)}\left(f,(g, h)^{\{a}\right)^{b\}}+\text { cycl. perm. }=0, \\
& \Delta^{\{a}(f, g)^{b\}}=\left(\Delta^{\{a} f, g\right)^{b\}}+(-1)^{\epsilon(f)+1}\left(f, \Delta^{\{a} g\right)^{b\}}, \\
& V^{\{a}(f, g)^{b\}}=\left(V^{\{a} f, g\right)^{b\}}+(-1)^{\epsilon(f)+1}\left(f, V^{\{a} g\right)^{b\}} .
\end{aligned}
$$

In the triplectic quantization schemes [6, 7] much stronger compatibility conditions are fulfilled, namely, instead of (2) and (7), one requires, respectively

$$
\Delta^{a} V^{b}+V^{a} \Delta^{b}=0, \quad V^{a}(f, g)^{b}=\left(V^{a} f, g\right)^{b}+(-1)^{\epsilon(f)+1}\left(f, V^{a} g\right)^{b} .
$$

The partition function in the triplectic formalism is defined by the expression

$$
Z=\int[d v][d \lambda] \mathrm{e}^{\frac{i}{\hbar}[W(z)+X(z, \lambda)]},
$$

where $[d v]$ stands for the volume element, $W(z)$ is viewed as the quantum action of the theory, and $X(z, \lambda)$ is considered as gauge fixing term. $\lambda$ are additional auxiliary variables which simply become Lagrangian multipliers of the gauge constraints when $X$ depends on them only linearly. The partition function (9) is gauge independent if the following "quantum master equations" hold [7]:

$$
\left(\Delta^{a}+\frac{i}{\hbar} V^{a}\right) \mathrm{e}^{\frac{i}{\hbar} W}=0 \quad \Leftrightarrow \quad-i \hbar \Delta^{a} W+V^{a} W+\frac{1}{2}(W, W)^{a}=0
$$

as well as a similar equation for the gauge fixing functional $\left(\Delta^{a}-\frac{i}{\hbar} V^{a}+\ldots\right) \mathrm{e}^{\frac{i}{\hbar} X}=0$ (the dots indicate the extra terms that are required due to the variation of the variables $\lambda$ ). The (anti)BRST transformations are generated by the operators $\delta^{a}$,

$$
\delta^{a}=(W-X, \cdot)^{a}+2 V^{a}+i \hbar \Delta^{a} .
$$

An important feature of the triplectic algebra which was observed in Ref. [7] is the existence of some Lagrangian submanifold $\mathcal{M}_{0}$ on which the following $S p(2)$ invariant, even Poisson bracket can be defined, viz.

$$
\{u, w\}_{0} \equiv \epsilon_{a b}\left(u, V^{a} w\right)^{b} .
$$

This submanifold $\mathcal{M}_{0}$ is specified by the requirement that all the functions $u(x), v(x), w(x)$ on $\mathcal{M}_{0}$ should obey the following conditions:

$$
\left.(u, v)^{a}=0, \quad\left(u, V^{\{a} v\right)^{b\}}, w\right)^{c}=0 .
$$

In that case it can be checked, by the use of Eqs. (4), (5) and (7), that the operation (12) defines an even Poisson bracket on $\mathcal{M}_{0}$ obeying the following relations:

$$
\begin{gathered}
\{u, v\}_{0}=-(-1)^{\epsilon(u) \epsilon(v)}\{v, u\}_{0}, \\
\{u, v w\}_{0}=\{u, v\}_{0} w+(-1)^{\epsilon(u) \epsilon(v)} v\{u, w\}_{0}, \\
(-1)^{\epsilon(u) \epsilon(w)}\left\{\{u, v\}_{0}, w\right\}_{0}+\text { cycl. perm. }=0 .
\end{gathered}
$$

In the canonical versions [5, 6] of the $S p(2)$ covariant Lagrangian quantization schemes this construction yields a canonical Poisson bracket on the fields $\phi^{A}$ and $\bar{\phi}_{A}$, namely, $\left\{\phi^{A}, \bar{\phi}_{B}\right\}_{0}=\delta_{B}^{A}$. 
Some efforts were performed to get the possible restrictions on this Poisson bracket and to clarify its role in the triplectic formalism. First of all, in Ref. 11], it was established that it is possible to construct the triplectic algebra when $\mathcal{M}_{0}$ is a special type of Kähler manifold; then, the triplectic algebra with nondegenerate antibrackets was constructed on the space of differential forms (on the external algebra) of arbitrary Kähler manifolds [12]. In the latter case the vector fields $V^{a}$ may be interpreted as the holomorphic and antiholomorphic external differentials, while the whole supermanifold was equipped with an even Kähler structure (see also [13]).

In this note we establish two properties of the triplectic formalism, viz.

- the explicit realization of the triplectic algebra over an arbitrary flat Poisson manifold (i.e. the Poisson manifold equipped with flat connection respecting Poisson bracket) is given;

- the triplectic formalism is equipped with an even symplectic structure.

The suggested structures seem to be important

- for the construction of an integration measure in the triplectic formalism, and

- for establishing a direct link between the Hamiltonian [14 and the Lagrangian [5, 6, 7] BRSTantiBRST invariant quantization schemes;

these are problems which, despite of some efforts on that subject 15, are still to be solved.

In our consideration we assume, for the simplicity, that $\mathcal{M}_{0}$ is a Poisson manifold, though in the BRST quantization formalisms it necessarily contains also Grassmannian degrees of freedom (e.g., ghosts/antighosts). Of course, the transport of the proposed constructions to supermanifolds is straightforward.

Poisson bracket and $V^{a}$ fields. Let us consider a Poisson manifold $\left(\mathcal{M}_{0},\{\cdot, \cdot\}_{0}\right)$ where the (even) Poisson bracket obeying Eqs. (14) is given by

$$
\{f(x), g(x)\}_{0}=\frac{\partial f(x)}{\partial x^{i}} \omega^{i j}(x) \frac{\partial g(x)}{\partial x^{j}}, \quad \omega^{i j}=-\omega^{j i}, \quad \omega^{i j}{ }_{, l} \omega^{l k}+\text { cycl. perm. }=0 .
$$

Then we consider the superspace $\mathcal{M}$ parametrized by the coordinates $x^{i}$ and $\theta_{i a},\left(\epsilon\left(\theta_{i a}\right)=\epsilon\left(x^{i}\right)+1=1\right)$, where $\theta_{i a}$ ("antifields") are transformed as $\partial / \partial x^{i}$ under reparametrizations of the base manifold $\mathcal{M}_{0}$ with coordinates $x^{i}$ ("fields"). In Darboux coordinates one has $x^{i}=\left(\phi^{A}, \bar{\phi}_{A}\right), \theta_{i a}=\left(\phi_{A a}^{*}, \varepsilon_{a b} \pi^{A b}\right)$.

On $\mathcal{M}$ we introduce the triplet of functions $S_{a b}$ defining an irreducible second rank $S p(2)$ tensor,

$$
S_{a b}=\frac{1}{6} \theta_{i a} \omega^{i j}(x) \theta_{j b}, \quad S_{a b}=S_{b a},
$$

and the following pairs of $\Delta$-operators and antibrackets transforming as $S p(2)$ doublets,

$$
\Delta^{a}=\nabla_{i} \frac{\partial_{l}}{\partial \theta_{i a}}+\frac{\partial \rho}{\partial x^{i}} \frac{\partial_{l}}{\partial \theta_{i a}}, \quad(f, g)^{a}=\nabla_{i} f \frac{\partial_{l} g}{\partial \theta_{i a}}+(-1)^{\epsilon(f)} \frac{\partial_{l} f}{\partial \theta_{i a}} \nabla_{i} g
$$

where $\nabla_{i}$ corresponds to the flat symmetric connection $\Gamma_{i j}^{k}(x)$ which respects the Poisson bracket (15) [16]:

$$
\nabla_{i} \equiv \frac{\partial}{\partial x^{i}}+\Gamma_{i j}^{k}(x) \theta_{k a} \frac{\partial}{\partial \theta_{j a}}: \quad \partial_{k} \omega^{i j}+\Gamma_{k l}^{i} \omega^{l j}+\omega^{i l} \Gamma_{l k}^{j}=0, \quad \Gamma_{i j}^{k}=\Gamma_{j i}^{k}, \quad\left[\nabla_{i}, \nabla_{j}\right]=0,
$$

while $\rho$ obeys the condition $\partial_{i} \omega^{i j}=\omega^{j i} \partial_{i} \rho$ (for nondegenerate Poisson brackets it yields the expression $\left.\rho=-\frac{1}{2} \log \operatorname{det} \omega^{i j}\right)$. When the Poisson bracket is nondegenerate, the flat connection exists, at least locally, due to the Darboux theorem, since in canonical coordinates one can choose the trivial connection which is obviously flat [17, 18]. Let us now introduce the (nilpotent) vector fields $V_{a}$ being generated by the antibrackets through the irreducible second rank $S p(2)$ tensor $S_{a b}$ according to:

$$
V_{a}=\left(S_{a b}, \cdot\right)^{b}=-\frac{1}{2} \theta_{i a} \omega^{i j} \nabla_{j} .
$$

These fields can also be represented in the form (no summation)

$$
V_{a}=3\left(S_{11}+S_{22}, \cdot\right)^{a}=6(-1)^{a}\left(S_{12}, \cdot\right)_{a}
$$


i.e., these vector fields are anti-Hamiltonian with respect to both antibrackets, and they obey the conditions (8). Hence they form, together with the antibrackets $(\cdot, \cdot)^{a}$ and the $\Delta^{a}$ - operators (17), the triplectic algebra (11), (3) - (6) and (8).

According to that procedure we constructed an explicit realization of the triplectic algebra on $\mathcal{M}$ with an arbitrary flat Poisson manifold $\mathcal{M}_{0}$.

$U^{a}$ fields. Let us now introduce particularly important realizations of anti-Hamiltonian vector fields generated by a $S p(2)$ scalar function $S_{0}$ according to

$$
U^{a}=\left(S_{0}, \cdot\right)^{a}: \quad S_{0}=\frac{1}{2} \varepsilon^{a b} \theta_{i a} g^{i j}(x) \theta_{j b}, \quad \epsilon\left(S_{0}\right)=0, \quad g^{i j}=g^{j i},
$$

with some (graded) symmetric tensor $g^{i j}$. Substituting these expressions into (12) yields a Poisson bracket which vanishes identically on $\mathcal{M}_{0}$ (even without requiring their nilpotency).

Such vector fields $U^{a}$ appear within the modified triplectic quantization [9]. In that case they are required to obey also the conditions

$$
U^{\{a} U^{b\}}=0, \quad V^{\{a} U^{b\}}+U^{\{a} V^{b\}}=0, \quad \Delta^{\{a} U^{b\}}+U^{\{a} \Delta^{b\}}=0,
$$

which are equivalent to the following ones,

$$
\left(S_{0}, S_{0}\right)^{a}=0, \quad V^{a} S_{0}=0, \quad \Delta^{a} S_{0}=0 .
$$

Now, it is convenient to introduce $I_{j}^{i}(x)=g_{j k} \omega^{i k}$, where $g^{i j}=\omega^{i k} g_{k l} \omega^{l j}$, and to interpret $g_{i j}$ as a "metric tensor" on $\mathcal{M}_{0}$. In that case Eqs. 23) imply the following conditions for $I_{j}^{i}$ :

$$
I_{i ; j}^{k}-I_{j ; i}^{k}=0, \quad I_{j}^{n} I_{n ; k}^{i}-I_{k}^{n} I_{n ; j}^{i}=0
$$

where the semicolon ";" denotes the covariant derivative. Hence, the Nijenhuis tensor $N_{j k}^{i}(I)$ of $I_{j}^{i}$ vanishes:

$$
N_{j k}^{i}(I) \equiv I_{j}^{n} I_{n ; k}^{i}-I_{k}^{n} I_{n ; j}^{i}-I_{n}^{i}\left(I_{j ; k}^{n}-I_{k ; j}^{n}\right)=0 .
$$

The latter equations can be resolved if one requires $\hat{I}$ to be an almost complex structure, $\hat{I}^{2}=-\mathrm{id}$, i.e., when $\mathcal{M}_{0}$ is a Kähler manifold.

So, on Kähler manifolds one can consistently formulate not only the triplectic algebra, but also the modified triplectic algebra.

Even Symplectic Structure. When the Poisson bracket (15) is non-degenerate, the superspace $\mathcal{M}$ can be equipped with both the even symplectic structure and the corresponding non-degenerate Poisson bracket,

$$
\begin{aligned}
\Omega=d z^{\mu} \Omega_{\mu \nu} d z^{\nu} & =\omega_{i j} d x^{i} \wedge d x^{j}+\alpha^{-1} \omega^{i j} D \theta_{i a} \wedge D \theta_{j}^{a}, \\
\{f(z), g(z)\} & =\left(\nabla_{i} f\right) \omega^{i j}\left(\nabla_{j} g\right)+\alpha \frac{\partial_{r} f}{\partial \theta_{i}^{a}} \omega_{i j} \frac{\partial_{l} g}{\partial \theta_{j a}},
\end{aligned}
$$

where $\alpha$ is an arbitrary constant, and $D \theta_{i a}=d \theta_{i a}-\Gamma_{i j}^{k} \theta_{k a} d x^{j}$. The proposed two-form $\Omega$ is closed (and, hence, the Jacobi identity for the Poisson bracket holds) due to the flatness of the symplectic connection and the equality $\omega^{i j} D \theta_{i a} \wedge D \theta_{j}^{a}=d\left(\theta_{i a} \omega^{i j} D \theta_{j}^{a}\right)$.

Using the even Poisson bracket as introduced above we can define the analogue of the Liouville measure on symplectic (super)surfaces $\Upsilon$ (and, as a consequence, on the whole superspace $\mathcal{M}$ ) defined by the help of the equations $f^{\mu}\left(x, \theta_{a}\right)=0$ (cf., Ref. 19]) as follows:

$$
\mathcal{D}_{\Upsilon}\left(x, \theta \mid f^{\mu}\right)=\sqrt{\operatorname{Ber}\left\{f^{\mu}, f^{\nu}\right\}} \delta\left(f^{\mu}\right) \mathcal{D}_{0}, \quad \mathcal{D}_{0}=\sqrt{\operatorname{Ber} \Omega_{\mu \nu}}=\alpha\left(\operatorname{det} \omega_{i j}\right)^{3 / 2} .
$$

These measures are invariant under supercanonical transformations of the Poisson bracket (26), as well as under smooth deformations of the surface $\Upsilon$ [19].

Note, that the antibrackets (17) may be connected with the proposed even symplectic structure as follows: $\Omega_{a}=\alpha \mathcal{L}_{a} \Omega$, where $\mathcal{L}_{a}$ denotes the Lie derivative along the vector field $V^{a}$, while the closed 
two-forms $\Omega_{a} \equiv d x^{i} \wedge D \theta_{i a}=d x^{i} \wedge d \theta_{i a}$, are pseudo-inverse to the antibrackets (17). The (super)group of transformations preserving simultaneously the Poisson bracket $\{\cdot, \cdot\}$ and the antibrackets $(\cdot, \cdot)^{a}$ is infinite-dimensional, due to the degeneracy of the antibrackets (while the group of transformations preserving simultaneously even and odd symplectic structures is necessarily finite-dimensional [3]). At this stage it appears as a natural desire to establish, by the use of the symplectic structure (26), a direct link between the $S p(2)$ invariant Lagrangian and Hamiltonian BRST formalisms. Furthermore, one may tend to use the measure (27) in the path integral of the triplectic formalism.

Discussion and summary. In this note we made some observations on the formal structure of the triplectic formalism in its "strong" and modified version.

First, we established that the vector fields $V^{a}$ appearing in the triplectic formalism can be represented as anti-Hamiltonian vector fields generated by the Poisson bracket through an irreducible second rank $S p(2)$ tensor. This allowed us to give a realization of the triplectic algebra, in an arbitrary coordinate system, by using a flat Poisson connection. Note, that the flat connection respecting Poisson bracket was used by I. A. Batalin and I. V. Tyutin for the formulation of coordinate-free scheme of deformation quantization [17]. The symmetric connections which respect the symplectic structure are widely known under a nickname "Fedosov connections" [16] due to Fedosov's works on globally defined deformation quantization [21]. These connections were recently found to be the natural objects in the Hamiltonian BRST quantization as well [22]. We have also shown that, requiring additionally the base Poisson manifold to possess a Kähler structure, allows to equip the triplectic algebra with additional vector fields $U^{a}$, appearing in modified triplectic quantization. These operators are responsible for the gauge fixing with a separation into "physical variables" and their "momenta".

Second, we have found that the triplectic algebra can be equipped by an even symplectic structure. This symplectic structure could be used for the construction of an integration measure in the triplectic formalism, as well as for establishing a direct link between Lagrangian and Hamiltonian BRST quantization

schemes. Note, that the existence of the even symplectic structure looks to be a natural one in the the superfield formulation of the BRST quantization [20, 23]. The symplectic structure $\Omega$ and the odd two-forms $\Omega_{a}$ could be extracted from the closed superfield two-form $\omega(\Phi)_{i j} d \Phi^{i} \wedge d \Phi^{j}$, where $\Phi^{i}=$ $x^{i}+\omega^{i j}\left(\eta^{a} \theta_{j a}+\eta^{1} \eta^{2} y_{j}\right), \epsilon\left(\eta^{a}\right)=1$ and $y_{i}$ are auxiliary fields denoting, in the flat case, sources and Lagrangian multipliers $y_{i}=\left(\lambda^{A}, J_{A}\right)$ [23].

In our opinion, it seems to be mysterious, that just the BRST/antiBRST invariant scheme includes, in its most symmetric (triplectic) formulation, all the ingredients appearing in various quantization formalisms. It might be assumed, that within that framework it would be possible to develop a complete (or universal) quantization scheme containing, as special limits, all existing methods, both BRST and non-BRST, Lagrangian and Hamiltonian ones.

Acknowledgements: The authors thank A. Karabegov for several discussions on Fedosov geometry, and I. V. Tyutin for criticism and useful remarks. P.L. and A.N. acknowledge the hospitality of NTZ at the Center of Advanced Study of Leipzig University and financial support by the Saxonian Ministry of Fine Arts and Sciences, which made this collaboration possible. The work of P.L. was also supported under the projects RFBR 99-02-16617, INTAS 99-0590, RFBR-DFG 99-02-04022 and the Fundamental Sciences Grant E00-3.3-461 of the Russian Ministry of Education. The work of A.N. was also supported under the INTAS project 00-A1-0262.

\section{References}

[1] I.A. Batalin and G.A. Vilkovisky, Phys. Lett. 102B (1981) 27; Phys. Rev. D28 (1983) 2567 [E: D30 (1984) 508]; Nucl. Phys. B234 (1984) 106.

[2] E. Witten, Mod. Phys. Lett. A 5 (1990) 487;

O. M. Khudaverdian and A. P. Nersessian, Mod. Phys. Lett. A 8 (1993) 2377; J. Math. Phys. 37 (1996) 3713.

A. Schwarz, Commun. Math. Phys. 155 (1993) 249; ibid. 158 (1993) 373.

I.A. Batalin and I.V. Tyutin, Int. J. Mod. Phys. A8 (1993) 2333; Mod. Phys. Lett. A8 (1993) 3673; ibid. A9 (1994) 1707. 
H. Hata and B. Zwiebach, Ann. Phys.(N.Y.) 322 (1994) 131.

J. Alfaro and P.H. Damgaard, Phys. Lett. B334 (1994) 369.

[3] O. M. Khudaverdian, J. Math. Phys. 32 (1991), 1934 (preprint UGVA-DPT 1989 / 05 - 613).

[4] O. M. Khudaverdian and R. L. Mkrtchian, Lett. Math. Phys. 18, 229 (1989),

O. M. Khudaverdian, Commun. Math. Phys. 198, 591 (1998); "Semidensities on odd symplectic manifolds", [math/DG/0012256]

[5] I.A. Batalin, P.M. Lavrov and I.V. Tyutin, J. Math. Phys. 31 (1990) 1487; ibid. 32 (1990) 532; ibid. 32 (1990) 2513.

[6] I.A. Batalin and R. Marnelius, Phys. Lett. B 350 (1995) 44.

[7] I. A. Batalin, R. Marnelius and A. M. Semikhatov, Nucl. Phys. B446 (1995), 249.

[8] M. Henneaux, Phys. Lett. B 282 (1992) 372.

P. Gregoire and M. Henneaux, Phys. Lett. B 277 (1992) 459; Commun. Math. Phys. 157 (1993) 279.

A. Nersessian and P. H. Damgaard, Phys. Lett. B355 (1995) 150.

I.A. Batalin and R. Marnelius, Nucl. Phys. B 465 (1996) 521.

M. A. Grigoriev and A. M. Semikhatov, Phys. Lett. B 417 (1998) 259.

M. A. Grigoriev, Phys. Lett. B 458 (1999) 499.

[9] B. Geyer, D.M. Gitman and P.M. Lavrov, Mod. Phys. Lett. A14 (1999) 661.

[10] B. Geyer, P.M. Lavrov and D. Mülsch, J. Math. Phys. 40 (1999) 674; ibid. 40 (1999) 6189;

B. Geyer and D. Mülsch, J. Math. Phys. 41 (2000) 7304.

[11] M. A. Grigoriev and A. M. Semikhatov, Theor. Math. Phys. 124 (2000) 1157.

[12] S. Bellucci and A. Nersessian, "Kähler geometry and SUSY mechanics", hep-th/0103005.

[13] A. P. Nersessian, Theor. Math. Phys. 96 (1993), 866; JETP Lett. 58 (1993) 66; Lecture Notes in Physics 524 (1997) 90; hep-th/9811110.

[14] I. A. Batalin, P. M. Lavrov and I. V. Tyutin, J. Math. Phys.31 (1990) 6; ibid. 31 (1990) 2708.

P. Gregoire and M. Henneaux, Phys. Lett. B 277 (1992) 459; J. Phys. A 26 (1993) 6073; Commun. Math. Phys. 157 (1993) 279.

[15] G. V. Grigorian, R. P. Grigorian and I. V. Tyutin, Nucl. Phys. B 379 (1992) 304.

F. De Jonghe, Phys. Lett. B 316 (1993) 503.

I. V. Tyutin and S. S. Shahverdiyev, Theor. Math. Phys. 110 (1997) 109.

[16] I. Gelfand, V. Retakh and M. Shubin, Adv. Math. 136 (1998) 104, dg-ga/9707024

[17] I. A. Batalin and I. V. Tyutin, Nucl. Phys. B 345 (1990) 645.

[18] B. V. Fedosov and A. V. Karabegov, private communication.

[19] O. M. Khudaverdian, A. S. Schwarz and Y. S. Tyupkin, Lett. Math. Phys. 5, 517 (1981).

[20] I. A. Batalin, K. Bering and P. H. Damgaard, Nucl. Phys. B 515 (1998) 455; Phys. Lett. B 446 (1999) 175.

M. A. Grigoriev and P. H. Damgaard, Phys. Lett. B 474 (2000) 323.

[21] B. V. Fedosov, J. Diff. Geom. 40 (1994) 213.

[22] M. A. Grigorev and S. L. Lyakhovich, "Fedosov deformation quantization as a BRST theory," hepth/0003114.

I. A. Batalin, M. A. Grigoriev and S. L. Lyakhovich, "Star product for second class constraint systems from a BRST theory," hep-th/0101089.

[23] P. M. Lavrov, Phys. Lett. B 366 (1996) 160; Theor. Math. Phys. 107 (1996) 602.

P. M. Lavrov and P. Yu. Moshin, "Superfield Lagrangian quantization with extended BRST symmetry," Phys. Lett. B (in press) hep-th/0102106. 